\title{
Impact of Packet Size on Performance of TCP traffic with Small Router buffers
}

\author{
Bo Zhang ${ }^{1, a}, \mathrm{Yu} \mathrm{Li}^{2}$ and Yanlei Liang ${ }^{2}$ \\ ${ }^{1,2}$ Faculty of Science and Technology, Communication University of China, China
}

\begin{abstract}
In this paper, we research the impact of packet size on the performance of TCP traffic with small router buffers. First of all, we established a simple model which had two TCP flows, combined with the queue management mechanism in a router and analyzed the effect on TCP packet loss performance of variable packet sizes. Secondly, the corresponding network topology was established based on the NS2 simulation software. And the correctness of the model was verified by experiments.
\end{abstract}

\section{Introduction}

Network technology develops rapidly and the scale of the Internet continues to expand. It put forward higher requirements on the network quality of service, and as the router is a hub to connect the different network, its performance directly determines the quality of service in network communication. The main performance indicators of the router include the throughput, delay, utilization and packet loss rate and so on. The router buffer is essential for network data exchange. The buffer can reduce the packet loss by absorbing the burst traffic, but it also causes the queuing delay and jitter. So, how to set the buffer size to make various performance parameters of the network achieve the best compromise will be a very important issue. It has been researched for a few years and some different conclusions are obtained. However, until now, there is still no unified conclusion on the research of router buffer requirement.

Currently commercial router buffer is still in accordance with the rule-of thumb [1] to design. It was proposed by Villamizar and song in 1994, namely B = $\overline{R T T} \times C$, where $B$ was the size of the buffer, $R T T$ was the average round-trip time of a TCP connection, $C$ was the bandwidth of the bottleneck link. But it was questioned by the Stanford Rule [2]. It proposed that if the number of TCP flows in the network was large enough, the transmission of TCP flows was tends to the nonsynchronization, and buffer size could be reduced to $1 / \sqrt{N}$ times, where $N$ was the number of the long TCP flows. Through extensive researches, some other rules had been put forward, like buffer sizing for congested Internet links [3] and tiny buffer rule [4]. It was pointed out that buffer size could be further decreased, even about dozens of packet groups would be able to meet the demand. Obviously, with the increasing of multimedia services in future network, the demand for bandwidth is increasing, and the rapid development of optical network determines the importance of the research of small buffer.

At the same time in studying the small buffer, it found that the network packet size was a very important factor for the performance of a router. Different packets sizes had different effects on the packet loss rate and the throughput. According to the needs of the link layer, usually in the Internet, packet size has been clearly defined, such as Ethernet. In the Ethernet the maximum packet size is 1500bytes [5]. However, a lot of applications (such as VoIP applications) usually use a small packet flow in order to minimize the delay time [6]. In another paper [7], the authors presented the result of simulation experiment on wireless IPv6 network by implementing TCP Vegas with the packet spacing adaption. Packet spacing improved the performance of TCP Vegas. In the simulation experiments, it controlled the flow packet interval uniform to minimize the burstiness of flow at the bottleneck link. Different sizes of FTP packets were sent in a wireless IPv6 environment. The experimental results showed that the data packets of different sizes leaded to different network performance. When FTP packet size was set 512bytes, it had smaller packet loss rate, lower queue delay, higher link utilization. Therefore, when the packet was transmitted in wireless IPv6 network, they suggested that the packet size is 512 bytes. However, no more useful information in papers suggested that the impacts of different packet sizes on the TCP flow were not fair. In order to study the effects of different packet sizes on the performance of TCP, we have done some research.

The rest of the paper is organized as follows. In Section II, we introduced the theoretical model to analyze the impact of packet size. In Section III, we made simulation and observed the performance impact of various packet sizes with different buffer capacity. We analyzed the experimental phenomenon and discussed its nonaccidental. We summarized our work and obtained the

a Corresponding author: zhangbo86108@163.com 
experimental conclusions, and pointed out directions for future work in Section IV.

\section{Theoretical model}

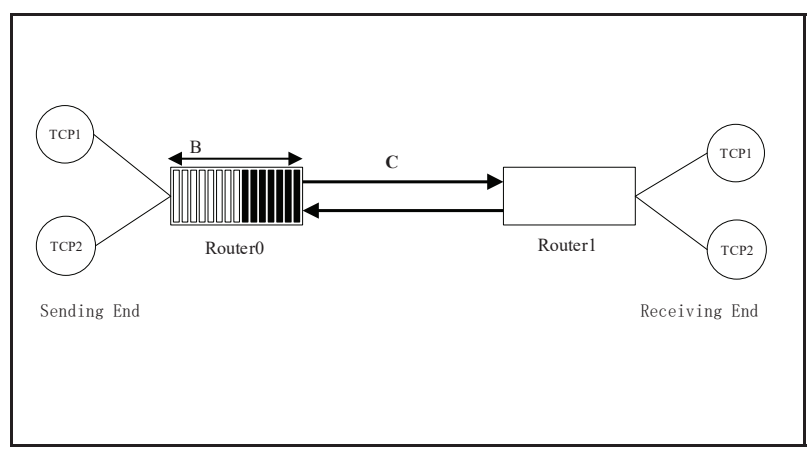

Figure 1. Theory Architecture of the Topology

As shown in Figure 1, we set up a simple dumbbell topology. We establish two TCP flow links. Among them, the bandwidth of bottleneck link is $C_{\text {out }}$, the bandwidth of each TCP flow access link is $C_{i n}$. Assume the packets size of first flow TCP is $l_{1}$, the other is $l_{2}$, and $l_{1}>l_{2}$. At the same time we assumed some related parameters as shown in Table 1.

Table 1. The Presentations of Parameters

\begin{tabular}{|c|c|}
\hline Parameters & Presentation \\
\hline$d_{1}$ & The amount of TCP1 loss packet \\
\hline$d_{2}$ & The amount of TCP2 loss packet \\
\hline$N_{1}$ & The amount of TCP1 packet \\
\hline$N_{2}$ & $\begin{array}{c}\text { The probability of a TCP1 packet reaching } \\
\text { to Router0 }\end{array}$ \\
\hline$P_{1}$ & $\begin{array}{c}\text { The probability of a TCP2 packet reaching } \\
\text { to Router0 }\end{array}$ \\
\hline$P_{2}$ & The probability of a TCP1 packet lost \\
\hline$p_{1}$ & The probability of a TCP2 packet lost \\
\hline$p_{2}$
\end{tabular}

In the paper [8], it was pointed out that the router buffer capacity had a very important impact on the arrival process of the TCP flows. If the buffers was too large, it could cause the synchronization between the TCP flows, which could cause a burst. But when the buffer became small, the distribution of the TCP flows could be approximated by the Poisson distribution. For us, the buffer was very small in experiments, so it could be considered that the TCP packets arrival obeyed Poisson distribution.

In our model, we assume that arrival rate of TCP1 is $\lambda_{1}$, arrival rate of TCP2 is $\lambda_{2}, \lambda_{1}=\lambda_{2}=\lambda$, and the rate of flow packets from the router to the bottleneck link is $C_{\text {out }}$. According to the length of router queue management strategy of Drop Tail, when the router queue is full, then the arrival packets will be discarded. Assuming the header packets in router buffer queue is a packet of TCP1, then the time that packet transferred from the router to the bottleneck link is:

$$
t_{1}=\frac{l_{1}}{C_{\text {out }}}
$$

Similarly, if this packet is TCP2's packet, then the time that packet from the router to the bottleneck link is:

$$
t_{2}=\frac{l_{2}}{C_{\text {out }}}
$$

Because of $l_{1}>l_{2}, t_{1}>t_{2}$.

Assuming that the router buffer capacity is full, in a very short period of time $t, t<t_{2}<t_{1}$, the probability that there is at least one TCP flow packet arriving to the router at this time according to the principle of Poisson distribution is:

$$
P=1-\frac{(\lambda t)^{0} e^{-\lambda t}}{0 !}=1-e^{-\lambda t}
$$

At the same time, $P$ also denotes the probability of the packet lost in the time $t$.

According to the Equation (3), the probability of a TCP1 or a TCP2 packet lost can be expressed in the following Equation:

$$
P_{1}=1-e^{-\lambda_{1} t}, \quad P_{2}=1-e^{-\lambda_{2} t}
$$

Furthermore, the ratio of the probability of a TCP1 packet lost to the probability of a TCP2 packet lost $R_{p}$ was:

$$
R_{p}=\frac{P_{1}}{P_{2}}=\frac{1-e^{-\lambda_{1} t}}{1-e^{-\lambda_{2} t}}
$$

In the period of time $t$, it is equal between the traffic of TCP1 and the traffic of TCP2. So the ratio of the amount of TCP1 to the amount of TCP2 $R_{N}$ was:

$$
R_{N}=\frac{N_{1}}{N_{2}}=\frac{\frac{s}{l_{1}}}{\frac{s}{l_{2}}}=\frac{l_{2}}{l_{1}}
$$

The ratio of TCP1 packet loss rate to TCP2 packet loss rate $R_{\text {loss }}$ could be expressed in the following Equation:

$$
R_{\text {loss }}=\frac{\frac{d_{1}}{N_{1}}}{\frac{d_{2}}{N_{2}}}=\frac{d_{1}}{d_{2}} \times \frac{N_{2}}{N_{1}}
$$

Through the above analysis,

$$
\frac{d_{T C P 1}}{d_{T C P 2}} \approx \frac{P_{1}}{P_{2}}=R_{P}
$$

According to Equation (5) (6) (7) (8), $R_{\text {loss }}$ could be expressed as:

$$
R_{\text {loss }}=\frac{1-e^{-\lambda_{1} t}}{1-e^{-\lambda_{2} t}} \times \frac{l_{1}}{l_{2}}
$$
that:

According to Equation (9), it could be obviously found

$$
l_{1}>l_{2}, \quad R_{\text {loss }}>1
$$

That is to say when the packet size of TCP1 was larger than the packet size of TCP2, the probability of TCP1 packet loss rate was larger than the probability of TCP2 packet loss rate either. So it can be drew that it's more easily discarded when the packet size is large.

\section{Simulation and Findings}

\subsection{The experiment topology and settings}




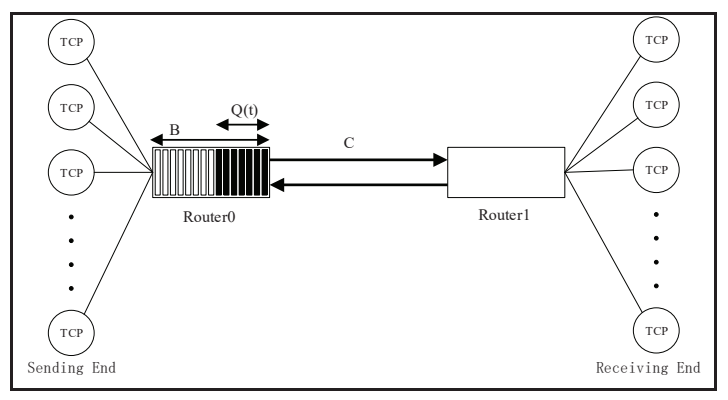

Figure 2. NS-2 simulation topology

In the last section of the theoretical analysis, we analyzed the simplest case, but there are a lot of TCP flows in the actual network, and there will be a certain impact between the flow and flow. So then we will validate our theoretical model through simulation experiments. The NS-2 simulation topology is shown in Fig.2. The classical dumbbell topology is commonly used. In the experiment, we used 100 TCP flows, so that each TCP flow can be approximately independent and not synchronized. Furthermore, we use TCP-Reno in all our simulations, consistent with the TCP version used in most of the Internet nowadays, and employ FIFO (First In First Out) queue with drop-tail queue management, which is commonly used in most routers today. We set the bottleneck link at $20 \mathrm{Mbps}$ and TCP access links at $5 \mathrm{Mbps}$ The propagation delay on the TCP access link is distributed between $[1,25] \mathrm{ms}$. The propagation delay on the bottleneck link ( $\mathrm{r} 0, \mathrm{r} 1)$ is 50ms; thus RTT (Round-Trip Time) vary between $104 \mathrm{~ms}$ and $200 \mathrm{~ms}$. All TCP sources start at random times between $[0,10] \mathrm{s}$. The simulation duration is $600 \mathrm{~s}$ and performance measurements are recorded after $100 \mathrm{~s}$, to allow for the stabilization of all TCP flows. In our experiment, we fix the buffer size, change the packet size of the TCP traffic, and measure the packet loss rate and throughput. According to the Stanford Rule, the best buffer size is $B=\frac{\overline{R T T} \times C}{\sqrt{n}} \approx 40 \mathrm{~KB}$, so we set the buffer size close to $40 \mathrm{~KB}$, including $20 \mathrm{~KB}, 30 \mathrm{~KB}$, $40 \mathrm{~KB}$ and $50 \mathrm{~KB}$. The packet size is between 200 byte and 1500 byte, and the step length is 100 byte.

\subsection{The Results}

Figure 3 shows the packet loss rate of the TCP traffic. Figure 4 shows the throughput of the TCP traffic.

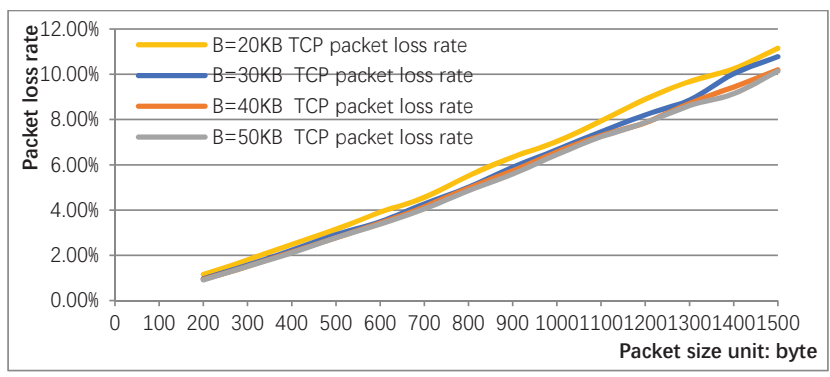

Figure 3. Packet loss rate of TCP traffic with different buffer sizes

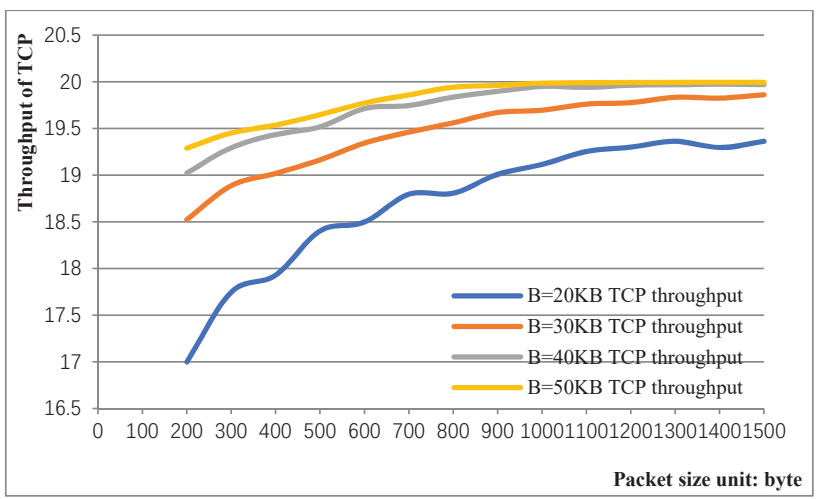

Figure 4. Throughput of TCP traffic with different buffer sizes

Figure 3 shows that when the buffer size is $20 \mathrm{~KB}, 30$ $\mathrm{KB}, 40 \mathrm{~KB}$ and $50 \mathrm{TCP}$, the packet loss rate of TCP traffic is changing with the various packet size. Obviously, with the increase of the buffer size, the packet loss rate of TCP is gradually decreased. For a fixed buffer size, the larger the packet size is, the greater the packet loss rate of the TCP traffic would be. This is consistent with the derivation of our theoretical model. Figure 4 shows the throughput of the TCP traffic with different buffer size and various packet size. It can be found longitudinally that when the buffer is set to $20 \mathrm{~KB}$, the queue space can not meet the requirements of the network, resulting in low throughput; when the buffer is $40 \mathrm{~KB}$, the queue space is large enough and the size of the buffer is not a factor limiting the throughput of TCP. At the same time, we can find that the impact of packet size on the throughput of TCP is not remarkable when the buffer is set reasonably. When the packet size increases gradually from the 200byte, the throughput of TCP flows increases slowly; when the packet size is up to1000byte, the throughput of TCP reaches a maximum and keep stable, so the TCP packet size is often set to 1000 byte in the experimental process.

According to the formula that the expression of the classic TCP throughput [9], the average throughput of a single flow can be expressed as:

$$
R_{L}=\frac{k L}{T \sqrt{p}}
$$

Assuming there are $n$ streams, the total throughput can be expressed as:

$$
R_{L}=n \times \frac{k L}{T \sqrt{p}}
$$

Where $k$ is constant, $T$ represents the average roundtrip time of TCP stream, $L$ denotes the length of the packet, and $p$ is the packet loss rate. For the same network environment, it can be seen that:

$$
R_{L} \propto \frac{L}{\sqrt{p}}
$$

It can be seen from Fig. 4 that the throughput of TCP traffic has a slow upward trend with the packet increasing, but this phenomenon deviated with our intuitive understanding. Theoretically, the throughput decreases with the increasing of the packet loss rate. In addition, from the Equation (13) it can be seen that the throughput has a relationship between packet length and packet loss rate, and the trend is changed by the packet length and packet loss rate. As seen from Fig.3, packet loss rate grows approximately in straight line with packet length. The growth rate of the packet is obviously faster than the 
square-root of the growth rate of the packet loss rate. So it has an upward trend of throughput of TCP traffic.

In conclusion, the larger size packets of the TCP stream are more likely to have behavior of packet loss, but large packets are more likely to get higher throughput, so a rational choice of packet size plays a critical role in performance of TCP flows.

\section{Conclusion}

This paper mainly studied the effect of TCP flow packet size on the performance of TCP. It included two aspects. In the first part, we established a simple model used two flows, which theoretically analyzed that bigger packets were likely to have greater packet loss rate. And in the second part, we carried out experiments on the network simulation platform to verify the conclusion of the network was applicable to multiple streams, mainly considering two aspects of TCP traffic: packet loss rate and throughput. The experimental results showed that the packet size had different effects on the packet loss rate and the throughput of the TCP stream. The larger the packet size was, the greater the packet loss rate and throughput would have. That was to say, it needed reasonable choice of the size of the packet in order to make the performance of TCP to reach the optimal. At the same time, it was pointed out that the impact of the unfairness of the packet size was only a qualitative study of the trend of the packet loss rate and throughput. And the paper only studied the unfairness effect on TCP performance by TCP packet size, and ignored open-loop flows in the network. These could be used as future research directions.

\section{ACKNOWLEDGMENT}

This work was supported in part by College Student Research and Career-creation Program of Beijing City.

\section{References}

1. C. Villamizar, C.Song, "High Performance TCP in ANSNET," ACM SIGCOMM Computer Communication Review, vol.24, no.5, Oct. 1994, pp. $45-60$

2. G. Appenzeller, I. Keslassy, N. McKeown, "Sizing Router Buffers," ACM SIGCOMM Computer Communication Review, vol.34, no.4, Oct. 2004, pp.281-292

3. A. Dhamdhere, H. Jiang, C. Dovrolis, "Buffer Sizing for Congested Internet Links," the 24th Annual Joint Conference of the IEEE Computer and Communications Societies, Miami, Florida, USA, Mar. 13-17, 2005, pp.1072-1083

4. M. Enachescu, Y. Ganjali, A.Goel, N. McKeown, T. Roughgarden, "Part Part III: Routers with very small buffers," ACM SIGCOMM Computer Communication Review, vol.35, no.2, July 2005, pp.83-89

5. J. Nieminen, J. Karvo, P. Lassila, M. Peuhkuri, "Impact of Heterogeneous Packet Sizes on Flow
Fairness," the 16th international conference on Telecommunications, Marrakech, Morocco, May 2527, 2009, pp.248-253

6. A. Lakshmikantha, C. Beck, and R. Srikant, \Impact of file arrivals and departures on buffer sizing in core routers," IEEE/ACM Transactions on Networking, pp. 347 -358, April 2011

7. B.S. Yew, B. L. Ong, and R. B. Ahmad, \Optimizing TCP Vegas performance with packet spacing and effect of variable FTP packet size over wireless IPv6 network," International Journal of Computer and Communication Engineering, vol. 6, pp. 89-97, February 2012

8. A. Vishwanath, V. Sivaraman, and D. Ostry, \How poisson is TCP traffic at short time-scales in a small buffer core network?," IEEE 3rd International Symposium on Advanced Networks and Telecommunication Systems, April 2009

9. M. Mathis, J. Semke, J. Mahdavi, T. Ott, "The Macroscopic Behaviors of the TCP Congestion Avoidance Algorithm," ACM SIGCOMM Computer Communication Review, 1997, pp.67-82. 